\title{
A New Bivariate Extended Generalized Inverted Kumaraswamy Weibull Distribution
}

\author{
Mahmoud Ragab $\mathbb{D}^{1,2,3}$ and Ahmed Elhassanein $\mathbb{D}^{4,5}$ \\ ${ }^{1}$ Information Technology Department, Faculty of Computing and Information Technology, King Abdulaziz University, \\ Jeddah 21589, Saudi Arabia \\ ${ }^{2}$ Mathematics Department, Faculty of Science, Al-Azhar University, Naser City, 11884 Cairo, Egypt \\ ${ }^{3}$ Centre of Artificial Intelligence for Precision Medicines, King Abdulaziz University, Jeddah 21589, Saudi Arabia \\ ${ }^{4}$ Department of Mathematics, College of Science, University of Bisha, Bisha, Saudi Arabia \\ ${ }^{5}$ Department of Mathematics, Faculty of Science, Damanhour University, Damanhour, Egypt \\ Correspondence should be addressed to Mahmoud Ragab; mragab@kau.edu.sa
}

Received 5 September 2021; Revised 1 November 2021; Accepted 25 November 2021; Published 27 January 2022

Academic Editor: David Carf

Copyright (c) 2022 Mahmoud Ragab and Ahmed Elhassanein. This is an open access article distributed under the Creative Commons Attribution License, which permits unrestricted use, distribution, and reproduction in any medium, provided the original work is properly cited.

\begin{abstract}
This article presents a new bivariate extended generalized inverted Kumaraswamy Weibull (BIEGIKw-Weibull) distribution with nine parameters. Statistical properties of the new distribution are discussed. Forms of copulas, moments, conditional moments, bivariate reliability function, and bivariate hazard rate function are derived. Maximum likelihood estimators are formulated. Simulation is conducted for three different sets of parameters to verify the theoretical results and to discuss the new distribution properties. The performance of the maximum likelihood method is investigated via Monte Carlo simulation depending on the bias and the standard error. Simulated lifetime data is used as an application of the new model.
\end{abstract}

\section{Introduction}

The inverse Weibull (IW) distribution is widely used because of its applicability in various fields, like medicine, statistics, engineering, physics, and fluid mechanics [1-11]. To enhance such distributions, researchers introduced new generators by supplementing shape parameters to the base line distribution. The inverted Kumaraswamy (IK) with two shape parameters has been derived by Abd AL-Fattah et al. [12]. To accommodate both monotonic and nonmonotonic failure rates, the IK distribution has been generalized to involve three shape parameters (GIKum) by Iqbal et al. [13]. A new version with five parameters (GIKw-W) has been introduced by Jamal et al. [14]. Although the univariate continuous models suit many types of data sets, they cannot be used to model dependent sets of data; therefore, a lot of efforts have been done to develop bivariate distributions. Muhammed [15] proposed a bivariate generalized Kumaras- wamy distribution. A bivariate inverse Weibull distribution has been developed by Mondal and Kundu [16]. Darwish and Shahbaz [17] formulated a bivariate transmuted Burr distribution; see also [18-25]. Most of the developed bivariate distributions have different shapes for the joint pdf and have singular part. In some cases, their joint probability distribution function can be expressed in compact forms. The maximum likelihood estimators cannot be expressed in explicit forms in most of the cases. Ganji et al. [26] generalized the method introduced by Alzaatreh et al. [27] to generate bivariate distributions with marginals having $T-$ $X$ families. Let $g_{X, Y}(x, y)$ be the pdf of the bivariate random variable $(X, Y)$, with $\left.\left.x \in a_{1}, b_{1}\right], y \in a_{2}, b_{2}\right],-\infty<a_{1}<b_{1}<\infty$ ,$-\infty<a_{2}<b_{2}<\infty$. Consider $F_{1}\left(G_{U}(u)\right)$ and $F_{2}\left(G_{V}(v)\right)$ be functions of the cdfs of a random variables $U$ and $V$, respectively, such that

(1) $F_{1}\left(G_{U}(u)\right) \in\left[a_{1}, b_{1}\right]$ and $F_{2}\left(G_{V}(v)\right) \in\left[a_{2}, b_{2}\right]$ 
(2) $F_{1}\left(G_{U}(u)\right)$ and $F_{2}\left(G_{V}(v)\right)$ are differentiable and monotonically nondecreasing functions

(3) $F_{1}\left(G_{U}(u)\right) \longrightarrow a_{1}$ as $u \longrightarrow-\infty, F_{1}\left(G_{U}(u)\right) \longrightarrow b_{1}$ as $u \longrightarrow \infty, F_{2}\left(G_{V}(v)\right) \longrightarrow a_{2}$ as $v \longrightarrow-\infty$, and $F_{2}$ $\left(G_{V}(v)\right) \longrightarrow b_{2}$ as $v \longrightarrow \infty$

The cdf of the random variable $(U, V)$ is given by

$F_{U, V}\left(F_{1}\left(G_{X}(u)\right), F_{2}\left(G_{Y}(z)\right)\right)=\int_{c_{1}}^{F_{1}\left(G_{X}(u)\right)} \int_{c_{2}}^{F_{2}\left(G_{Y}(v)\right)} g_{X, Y}(x, y) d x d y$.

In this paper, we introduce a new bivariate extended generalized inverted Kumaraswamy Weibull (BIEGIKwWeibull) distribution; its joint pdf is absolutely continuous, takes only one form with no singular parts, and offers different shapes for different values of parameters, and its hazard function shows different shapes. Almost all statistical quantities of the new distribution can be obtained in closed forms including the maximum likelihood estimators. The new model is developed using the new six parameter distribution that is more flexible with so favorable properties [28]. Theoretical properties of the proposed distribution including marginal distributions, copulas, moments, conditional moments, bivariate reliability function, and bivariate hazard function are computed. Theoretical properties are investigated via simulation. Monte Carlo simulation is used to discuss the goodness of fit and the availability of the maximum likelihood method. A real data application is presented that proves the applicability of the new distribution. The paper is organized as follows. The new distribution is formulated in Section 2. In Section 3, closed forms of moments are derived. Reliability and hazard function are computed in Section 4. Estimation is performed in Section 5. Simulation for different three sets of parameters is performed in Section 6. A real data application is discussed in Section 7. Conclusion is given in Section 8.

\section{Model Description}

A one-dimensional random variable $Z$ is said to have a GIKw-Weibull distribution if its cumulative distribution function (cdf) is given by

$$
F(z)=\left(1-\left\{1+\left(\left[1-e^{-\delta z^{\varphi}}\right]^{-\lambda}-1\right)^{-\gamma}\right\}^{-\alpha}\right)^{\beta}
$$

where $z>0, \alpha, \beta, \gamma, \delta, \lambda, \varphi>0$ are shape parameters [29]. Using (2) as a baseline distribution and $g_{X, Y}(x, y)=1+\delta_{1}$ $(1-x)+\delta_{2}(1-y)+2 \delta_{3}(1-x-y)$ in (1), where $\left.x, y \in 0,1\right]$, $\left.\delta_{1}, \delta_{2}, \delta_{3} \in-1,1\right],-1 \leq \delta_{1}+\delta_{3} \leq 1$, and $-1 \leq \delta_{2}+\delta_{3} \leq 1$, we formulate the following definition.

Definition 1. A bivariate random variable $(U, V)$ is said to be a BIEGIKw-Weibull random variable if its cumulative probability function (cdf) and probability density function (pdf) are given by

$$
\begin{aligned}
F_{U, V}(u, v)= & \left(1-\left\{1+\left(\left[1-e^{-\delta u^{\varphi}}\right]^{-\lambda}-1\right)^{-\gamma}\right\}^{-\alpha}\right)^{\beta} \\
& \times\left(1-\left\{1+\left(\left[1-e^{-\delta v^{\varphi}}\right]^{-\lambda}-1\right)^{-\gamma}\right\}^{-\alpha}\right)^{\beta} \\
& \cdot\left\{1+\left(\delta_{1}+\delta_{3}\right) \times\left(1-\left(1-\left\{1+\left(\left[1-e^{-\delta u^{\varphi}}\right]^{-\lambda}-1\right)^{-\gamma}\right\}^{-\alpha}\right)^{\beta}\right)\right. \\
& \left.+\left(\delta_{2}+\delta_{3}\right) \times\left(1-\left(1-\left\{1+\left(\left[1-e^{-\delta v^{\varphi}}\right]^{-\lambda}-1\right)^{-\gamma}\right\}^{-\alpha}\right)^{\beta}\right)\right\},
\end{aligned}
$$

$$
\begin{aligned}
& f_{U, V}(u, v)=(\alpha \beta \gamma \lambda \delta \varphi)^{2}(u v)^{\varphi-1} e^{-\delta\left(u^{\varphi}+v^{\varphi}\right)} \\
&\left(\left[1-e^{-\delta u^{\varphi}}\right]\left[1-e^{-\delta v^{\varphi}}\right]\right)^{-(\lambda+1)} \times \\
&\left\{\left(\left[1-e^{-\delta u^{\varphi}}\right]^{-\lambda}-1\right)\left(\left[1-e^{-\delta v^{\varphi}}\right]^{-\lambda}-1\right)\right\}^{-(\gamma+1)} \times \\
&\left\{\left(1+\left(\left[1-e^{-\delta u^{\varphi}}\right]^{-\lambda}-1\right)^{-\gamma}\right)\left(1+\left(\left[1-e^{-\delta v^{\varphi}}\right]^{-\lambda}-1\right)^{-\gamma}\right)\right\}^{-(\alpha+1)} \\
& \times\left\{\left(1-\left\{1+\left(\left[1-e^{-\delta u^{\varphi}}\right]^{-\lambda}-1\right)\right\}^{-\alpha}\right) \times\left(1-\left\{1+\left(\left[1-e^{-\delta v^{\varphi}}\right]^{-\lambda}\right.\right.\right.\right. \\
&\left.\left.\left.-1)^{-\gamma}\right\}^{-\alpha}\right)\right\}^{\beta-1} \times\left\{1+\left(\delta_{1}+\delta_{3}\right)(1-2\right. \\
&\left.\left(1-\left\{1+\left(\left[1-e^{-\delta u^{\varphi}}\right]^{-\lambda}-1\right)^{-\gamma}\right\}^{-\alpha}\right)^{\beta}\right)+\left(\delta_{2}+\delta_{3}\right)(1-2 \\
&\left.\left(1-\left\{1+\left(\left[1-e^{-\delta v^{\varphi}}\right]^{-\lambda}-1\right)^{-\gamma}\right\}^{-\alpha}\right)\right\},
\end{aligned}
$$

where $\left.\quad u, v>0, \alpha, \beta, \gamma, \delta>0, \delta_{1}, \delta_{2}, \delta_{3} \in-1,1\right],-1 \leq \delta_{1}+\delta_{3}$ $\leq 1$, and $-1 \leq \delta_{2}+\delta_{3} \leq 1$.

\section{Marginals and Moments}

Lemma 2. Let $(U, V)$ be a BIEGIKw-Weibull random variable with cdf and pdf given in (3) and (4). Then, the marginals are

$$
\begin{aligned}
F_{U}(u)= & \left(1-\left\{1+\left(\left[1-e^{-\delta u^{\varphi}}\right]^{-\lambda}-1\right)^{-\gamma}\right\}^{-\alpha}\right)^{\beta} \\
& \times\left\{1+\left(\delta_{1}+\delta_{3}\right)\left(1-\left(1-\left\{1+\left(\left[1-e^{-\delta u^{\varphi}}\right]^{-\lambda}-1\right)^{-\gamma}\right\}^{-\alpha}\right)\right\}\right. \\
F_{V}(v)= & \left(1-\left\{1+\left(\left[1-e^{-\delta v^{\varphi}}\right]^{-\lambda}-1\right)^{-\gamma}\right\}^{-\alpha}\right)^{\beta} \\
& \times\left\{1+\left(\delta_{2}+\delta_{3}\right)\left(1-\left(1-\left\{1+\left(\left[1-e^{-\delta \nu^{\varphi}}\right]^{-\lambda}-1\right)^{-\gamma}\right\}^{-\alpha}\right)^{\beta}\right)\right\}
\end{aligned}
$$

$$
\begin{aligned}
f_{U}(u)= & \alpha \beta \gamma \lambda \delta \varphi u^{\varphi-1} e^{-\delta u^{\varphi}}\left[1-e^{-\delta u^{\varphi}}\right]^{-(\lambda+1)}\left(\left[1-e^{-\delta u^{\varphi}}\right]^{-\lambda}-1\right)^{-(\gamma+1)} \\
& \times\left\{1+\left(\left[1-e^{-\delta u^{\varphi}}\right]^{-\lambda}-1\right)^{-\gamma}\right\}^{-(\alpha+1)} \\
& \times\left(1-\left\{1+\left(\left[1-e^{-\delta u^{\varphi}}\right]^{-\lambda}-1\right)^{-\gamma}\right\}^{-\alpha}\right)^{\beta-1} \\
& \times\left\{1+\delta_{1}+\delta_{3}-2\left(\delta_{1}+\delta_{3}\right)\left(1-\left\{1+\left(\left[1-e^{-\delta u^{\varphi}}\right]^{-\lambda}-1\right)^{-\gamma}\right\}^{-\alpha}\right)^{\beta}\right\},
\end{aligned}
$$




$$
\begin{aligned}
f_{V}(v)= & \alpha \beta \gamma \lambda \delta \varphi v^{\varphi-1} e^{-\delta v^{\varphi}}\left[1-e^{-\delta v^{\varphi}}\right]^{-(\lambda+1)}\left(\left[1-e^{-\delta v^{\varphi}}\right]^{-\lambda}-1\right)^{-(\gamma+1)} \\
& \times\left\{1+\left(\left[1-e^{-\delta v^{\varphi}}\right]^{-\lambda}-1\right)^{-\gamma}\right\}^{-(\alpha+1)} \\
& \times\left(1-\left\{1+\left(\left[1-e^{-\delta v^{\varphi}}\right]^{-\lambda}-1\right)^{-\gamma}\right\}^{-\alpha}\right)^{\beta-1} \\
& \times\left\{1+\delta_{2}+\delta_{3}-2\left(\delta_{2}+\delta_{3}\right)\left(1-\left\{1+\left(\left[1-e^{-\delta v^{\varphi}}\right]^{-\lambda}-1\right)^{-\gamma}\right\}^{-\alpha}\right)^{\beta}\right\} .
\end{aligned}
$$

For $\delta_{1}=\delta_{2}=-\delta_{3}$, we get the baseline distribution EGIKw-Weibull.

Copula function is commonly used to investigate the dependence between two random variables.

Definition 3 (see [30]). Let $(U, V)$ be a BIGIKw-Weibull random variable with cpf $F_{U, V}(u, v)$ and marginals $F_{U}(u)$ and $F_{V}(v)$, and then, its copula function can be defied as $F_{U, V}(u, v) \triangleq C(v, \phi)$, where $v=F_{U}(u), \phi=F_{V}(v)$, and $v, \phi$ $\epsilon(0,1)$ and the copula density function is defined as $c(v, \phi)$ $=\partial^{2} C(v, \phi) / \partial v \partial \phi$.

Using chain rule, we obtain

$$
\begin{aligned}
f_{U, V}(u, v) & =\frac{\partial^{2} F(u, v)}{\partial u \partial v}=\frac{\partial^{2} C\left(F_{U}(u), F_{V}(v)\right)}{\partial u \partial v} \\
& =\frac{\partial^{2} C(v, \phi)}{\partial v \partial \phi} \frac{\partial F_{U}(u)}{\partial u} \frac{\partial F_{V}(v)}{\partial v} \triangleq c(v, \phi) f_{U}(u) f_{V}(v) .
\end{aligned}
$$

Lemma 4. Let $(U, V)$ be a BIEGIKw-Weibull random variable with pdf and marginals given in (4), (7), and (8). Then, the copula density function is given by

$$
\begin{aligned}
c(v, \phi)= & \frac{1}{H\left(u, \delta_{1}\right) H\left(v, \delta_{2}\right)} \\
& \cdot\left\{1+\left(\delta_{1}+\delta_{3}\right) \times\left(1-2\left(1-\left\{1+\left(\left[1-e^{-\delta u^{\varphi}}\right]^{-\lambda}-1\right)^{-\gamma}\right\}^{-\alpha}\right)^{\beta}\right)\right. \\
& \left.+\left(\delta_{2}+\delta_{3}\right)\left(1-2\left(1-\left\{1+\left(\left[1-e^{-\delta v^{\varphi}}\right]^{-\lambda}-1\right)^{-\gamma}\right\}^{-\alpha}\right)^{\beta}\right)\right\} .
\end{aligned}
$$

Lemma 5. Let $(U, V)$ be a BIEGIKw-Weibull random variable with pdf and marginals given in (4), (7), and (8). Then, the conditional density functions are

$$
\begin{aligned}
f_{V}(u / v)= & \frac{\left.\alpha \beta \gamma \delta \varphi u^{\varphi-1} e^{-\delta u^{\varphi}}\left[1-e^{-\delta u^{\varphi}}\right)\right]^{-(\lambda+1)}}{H\left(v, \delta_{2}\right)} \\
& \times\left(1-\left[1-e^{-\delta u^{\varphi}}\right]^{\lambda}\right)^{-(\gamma+1)}\left\{1+\left(\left[1-e^{-\delta u^{\varphi}}\right]^{-\lambda}-1\right)^{-\gamma}\right\}^{-(\alpha+1)} \\
& \times\left(1-\left\{1+\left(\left[1-e^{-\delta u^{\varphi}}\right]^{-\lambda}-1\right)^{-\gamma}\right\}^{-\alpha}\right)^{\beta-1} \\
& \times\left\{1+\left(\delta_{1}+\delta_{3}\right)\left(1-2\left(1-\left\{1+\left(\left[1-e^{-\delta u^{\varphi}}\right]^{-\lambda}-1\right)^{-\gamma}\right\}^{-\alpha}\right)^{\beta}\right)\right.
\end{aligned}
$$

$$
\begin{aligned}
+\left(\delta_{2}+\right. & \left.\left.\delta_{3}\right)\left(1-2\left(1-\left\{1+\left(\left[1-e^{-\delta v^{\varphi}}\right]^{-\lambda}-1\right)^{-\gamma}\right\}^{-\alpha}\right)^{\beta}\right)\right\}, \quad(11) \\
f_{\frac{V}{U}}(v / u)= & \frac{\alpha \beta \gamma \lambda \delta \varphi v^{\varphi-1} e^{-\delta v^{\varphi}}\left[1-e^{-\delta v^{\varphi}}\right]^{-(\lambda+1)}}{H\left(u, \delta_{1}\right)}\left(1-\left[1-e^{\left.\left.-\delta v^{\varphi}\right]^{\lambda}\right)^{-\gamma-1}}\right.\right. \\
& \left.\times\left\{1+\left(1-e^{-\delta v^{\varphi}}\right]^{-\lambda}-1\right)^{-\gamma}\right\}^{-\alpha-1} \\
& \left.\times\left(1-\left\{1+\left(1-e^{-\delta v^{\varphi}}\right]^{-\lambda}-1\right)^{-\gamma}\right\}^{-\alpha}\right)^{\beta-1} \\
& \cdot\left\{1+\left(\delta_{1}+\delta_{3}\right)\left(1-2\left(1-\left\{1+\left(\left[1-e^{-\delta u^{\varphi}}\right]^{-\lambda}-1\right)^{-\gamma}\right\}^{-\alpha}\right)\right.\right. \\
& \left.+\left(\delta_{2}+\delta_{3}\right)\left(1-2\left(1-\left\{1+\left(\left[1-e^{-\delta v^{\varphi}}\right]^{-\lambda}-1\right)^{-\gamma}\right\}^{-\alpha}\right)^{\beta}\right)\right\}
\end{aligned}
$$

where

$$
\begin{aligned}
H(x, \delta)=1 & +\delta+\delta_{3} 3.7-2\left(\delta+\delta_{3}\right) \\
& \cdot\left(1-2\left(1-\left\{1+\left(\left[1-e^{-\delta x^{\varphi}}\right]^{-\lambda}-1\right)^{-\gamma}\right\}^{-\alpha}\right)^{\beta}\right) .
\end{aligned}
$$

Lemma 6. Let $(U, V)$ be a BIEGIKw-Weibull random variable whose conditionals are given in (11) and (13). Then, the conditional moments are given by

$$
\begin{aligned}
& m_{V}^{r}(v)=E\left(\frac{U^{r}}{V}=v\right)=\frac{1}{H\left(v, \delta_{2}\right)}\left\{\frac { 1 } { \delta ^ { r / \varphi } } \left(1+\delta_{1}+\delta_{2}+2 \delta_{3}\right.\right. \\
& -2\left(\delta_{2}+\delta_{3}\right)\left(1-2\left(1-\left\{1+\left(\left[1-e^{-\delta \psi^{\varphi}}\right]^{-\lambda}-1\right)^{-\gamma}\right\}^{-\alpha}\right)^{\beta}\right) \\
& \times \sum_{i, j, k=0}^{\infty} \sum_{l=0}^{\lambda(\gamma j+k)-1}(-1)^{l} \xi_{i, j, k}\left(\begin{array}{c}
\lambda(\gamma j+k)-1 \\
l
\end{array}\right) \frac{\Gamma((r / \varphi)+1)}{(l+1)^{(r / \varphi)+1}} \\
& \left.-\frac{2\left(\delta_{1}+\delta_{3}\right)}{\delta^{r / \varphi}} \sum_{i, j, k=0}^{\infty} \sum_{l=0}^{2 \lambda(\gamma j+k)-1}(-1)^{l} \xi_{i, j, k}\left(\begin{array}{c}
\lambda(\gamma j+k)-1 \\
l
\end{array}\right) \times \frac{\Gamma((r / \varphi)+1)}{(l+1)^{(r / \varphi)+1}}\right\}, \\
& m_{V / U}^{r}(u)=E\left(\frac{V^{r}}{U}=u\right)=\frac{1}{H\left(u, \delta_{1}\right)} \\
& \left\{\frac { 1 } { \delta ^ { r / \varphi } } \left(1+\delta_{1}+\delta_{2}+2 \delta_{3}-2\left(\delta_{2}+\delta_{3}\right)\right.\right. \\
& \left.\cdot\left(1-2\left(1-\left\{1+\left(\left[1-e^{-\delta u^{\varphi}}\right]^{-\lambda}-1\right)^{-\gamma}\right\}^{-\alpha}\right)^{\beta}\right)\right) \\
& \times \sum_{i, j, k=0}^{\infty} \sum_{l=0}^{\lambda(\gamma j+k)-1}(-1)^{l} \xi_{i, j, k}(\beta)\left(\begin{array}{c}
\lambda(\gamma j+k)-1 \\
l
\end{array}\right) \frac{\Gamma((r / \varphi)+1)}{(l+1)^{(r / \varphi)+1}} \\
& -\frac{2\left(\delta_{1}+\delta_{3}\right)}{\delta^{r / \varphi}} \sum_{i, j, k=0}^{\infty} \sum_{l=0}^{\lambda(\gamma j+k)-1}(-1)^{l} \xi_{i, j, k}(2 \beta) \\
& \left.\left(\begin{array}{c}
\lambda(\gamma j+k)-1 \\
l
\end{array}\right) \times \frac{\Gamma((r / \varphi)+1)}{(l+1)^{(r / \varphi)+1}}\right\}
\end{aligned}
$$

where

$$
\xi_{i, j, k}(\rho)=(-1)^{i+j} \lambda(\gamma j+k)\left(\begin{array}{c}
\rho \\
i
\end{array}\right)\left(\begin{array}{c}
\alpha i+j-1 \\
j
\end{array}\right)\left(\begin{array}{c}
\gamma j+k-1 \\
k
\end{array}\right) .
$$


$\gamma$ is a positive integer and $H$ is given by (13); for more details, see [28].

Lemma 7. Let $(U, V)$ be a BIEGIKw-Weibull random variable whose density function is given in (4). Then, the joint moments are given by

$$
\begin{aligned}
m_{r, s}= & E\left(U^{r} V^{s}\right)=\frac{\left(1+\delta_{1}+\delta_{2}+2 \delta_{3}\right)}{\delta^{r / \varphi} \delta^{s / \varphi}} \\
& \times\left\{\sum_{i, j, k=0}^{\infty} \sum_{l=0}^{\lambda(\gamma j+k)-1}(-1)^{l} \xi_{i, j, k}(\beta)\left(\begin{array}{c}
\lambda(\gamma j+k)-1 \\
l
\end{array}\right) \frac{\Gamma((r / \varphi)+1)}{(l+1)^{(r / \varphi)+1}}\right. \\
& \left.\times \sum_{o, p, q=0}^{\infty} \sum_{t=0}^{\lambda(\gamma p+q)-1}(-1)^{t} \xi_{i, j, k}(\beta)\left(\begin{array}{c}
\lambda(\gamma p+q)-1 \\
t
\end{array}\right) \frac{\Gamma((s / \varphi)+1)}{(t+1)^{(s / \varphi)+1}}\right] \\
& -\frac{2}{\delta^{r / \varphi} \delta^{s / \varphi}}\left[\sum_{i, j, k=0}^{\infty} \sum_{l=0}^{\lambda(\gamma j+k)-1}(-1)^{l} \xi_{i, j, k}(2 \beta)\left(\begin{array}{c}
2 \lambda(\gamma j+k)-1 \\
l
\end{array}\right) \frac{\Gamma((r / \varphi)+1)}{(l+1)^{(r / \varphi)+1}}\right. \\
& \left.\times \sum_{o, p, q=0}^{\infty} \sum_{t=0}^{\lambda(\gamma p+q)-1}(-1)^{t} \xi_{o, p, q}(\beta)\left(\begin{array}{c}
\lambda(\gamma p+q)-1 \\
t
\end{array}\right) \frac{\Gamma((s / \varphi)+1)}{(t+1)^{(s / \varphi)+1}}\right] \\
& -\frac{2\left(\delta_{2}+\delta_{3}\right)}{\delta^{r / \varphi} \delta^{s / \varphi}}\left[\sum_{i, j, k=0}^{\infty} \sum_{l=0}^{\lambda(\gamma j+k)-1}(-1)^{l} \xi_{i, j, k}(\beta)\left(\begin{array}{c}
\lambda(\gamma j+k)-1 \\
l
\end{array}\right) \frac{\Gamma((r / \varphi)+1)}{(l+1)^{(r / \varphi)+1}}\right. \\
& \left.\times \sum_{o, p, q=0}^{\infty} \sum_{t=0}^{\lambda(\gamma j+q)-1}(-1)^{t} \xi_{o, p, q}(2 \beta)\left(\begin{array}{c}
2 \lambda(\gamma p+q)-1 \\
t
\end{array}\right) \frac{\Gamma((s / \varphi)+1)}{(t+1)^{(s / \varphi)+1}}\right\},
\end{aligned}
$$

where $\gamma$ is a positive integer and $\xi_{i, j, k}(\rho)$ is given by Equation (15).

\section{Reliability and Hazard Functions}

Bivariate hazard function can be used to characterize bivariate distributions. It describes the failure characteristics of the individual variables and their joint failure behavior. Here, we compute the bivariate reliability function and the hazard function defined by Navarro [30].

Lemma 8. Let $(U, V)$ be a BIEGIKw-Weibull random variable whose cumulative and marginals are given in (3), (5), and (6), respectively. Then, its bivariate reliability function is given by

$$
R(u, v)=R_{1}(u, v)+R_{2}(u, v),
$$

where

$$
\begin{aligned}
R_{1}(u, v)= & -\left(1-\left\{1+\left(\left[1-e^{-\delta u^{\varphi}}\right]^{-\lambda}-1\right)^{-\gamma}\right\}^{-\alpha}\right)^{\beta} \\
& \times\left(1+\left(\delta_{1}+\delta_{3}\right)\left(1-\left\{1+\left(\left[1-e^{-\delta u^{\varphi}}\right]^{-\lambda}-1\right)^{-\gamma}\right\}^{-\alpha}\right)^{\beta}\right) \\
& -\left(1-\left\{1+\left(\left[1-e^{-\delta \nu^{\varphi}}\right]^{-\lambda}-1\right)^{-\gamma}\right\}^{-\alpha}\right)^{\beta} \\
& \times\left(1+\left(\delta_{2}+\delta_{3}\right)\left(1-\left\{1+\left(\left[1-e^{-\delta v^{\varphi}}\right]^{-\lambda}-1\right)^{-\gamma}\right\}^{-\alpha}\right)^{\beta}\right)
\end{aligned}
$$

$R_{2}(u, v)=(\alpha \beta \gamma \lambda \delta \varphi)^{2}(u v)^{\varphi-1} e^{-\delta\left(u^{\varphi}+v^{\varphi}\right)}\left(\left[1-e^{-\delta u^{\varphi}}\right]\left[1-e^{-\delta v^{\varphi}}\right]\right)^{-(\lambda+1}$

$$
\begin{aligned}
& \times\left(\left(\left[1-e^{-\delta u^{\varphi}}\right]^{-\lambda}-1\right)\left(\left[1-e^{-\delta \nu^{\varphi}}\right]^{-\lambda}-1\right)\right)^{-(\gamma+1)} \\
& \times\left\{\left(1+\left(\left[1-e^{-\delta u^{\varphi}}\right]^{-\lambda}-1\right)^{-\gamma}\right)\left(1+\left(\left[1-e^{-\delta \nu^{\varphi}}\right]^{-\lambda}-1\right)^{-\gamma}\right)\right\}^{-(\alpha+1)} \\
& \times\left\{\left(1-\left\{1+\left(\left[1-e^{-\delta u^{\varphi}}\right]^{-\lambda}-1\right)^{-\gamma}\right\}^{-\alpha}\right)\right. \\
& \left.\times\left(1-\left\{1+\left(\left[1-e^{-\delta \nu^{\varphi}}\right]^{-\lambda}-1\right)^{-\gamma}\right\}^{-\alpha}\right)\right\}^{\beta-1} \\
& \times\left\{1+\left(\delta_{1}+\delta_{3}\right)\left(1-2\left(1-\left\{1+\left(\left[1-e^{-\delta u^{\varphi}}\right]^{-\lambda}-1\right)^{-\gamma}\right\}^{-\alpha}\right)^{\beta}\right)\right. \\
& \left.+\left(\delta_{2}+\delta_{3}\right)\left(1-2\left(1-\left\{1+\left(\left[1-e^{-\delta v^{\varphi}}\right]^{-\lambda}-1\right)^{-\gamma}\right\}^{-\alpha}\right)^{\beta}\right)\right\}
\end{aligned}
$$

Lemma 9. Let $(U, V)$ be a BIEGIKw-Weibull random variable whose density and reliability functions are given in (4) and (17). Then, its bivariate hazard rate function is given by

$$
\begin{aligned}
& h(u, v)=\left\{(\alpha \beta \gamma \lambda \delta \varphi)^{2}(u v)^{\varphi-1} e^{-\delta\left(u^{\varphi}+v^{\varphi}\right)}\left(\left[1-e^{-\delta u^{\varphi}}\right]\left[1-e^{-\delta v^{\varphi}}\right]\right)^{-(\lambda+1)}\right. \\
& \times\left\{\left(\left[1-e^{-\delta u^{\varphi}}\right]^{-\lambda}-1\right)\left(\left[1-e^{-\delta v^{\varphi}}\right]^{-\lambda}-1\right)\right\}^{-(\gamma+1)} \\
& \times\left\{\left(1+\left(\left[1-e^{-\delta u^{\varphi}}\right]^{-\lambda}-1\right)^{-\gamma}\right)\left(1+\left(\left[1-e^{-\delta v^{\varphi}}\right]^{-\lambda}-1\right)^{-\gamma}\right)\right\}^{-(\alpha+1)} \\
& \times\left\{\left(1-\left\{1+\left(\left[1-e^{-\delta u^{\varphi}}\right]^{-\lambda}-1\right)^{-\gamma}\right\}^{-\alpha}\right)\right. \\
& \left.\times\left(1-\left\{1+\left(\left[1-e^{-\delta \varphi^{\varphi}}\right]^{-\lambda}-1\right)^{-\gamma}\right\}^{-\alpha}\right)\right\}^{\beta-1} \\
& \times\left\{1+\left(\delta_{1}+\delta_{3}\right)\left(1-2\left(1-\left\{1+\left(\left[1-e^{-\delta u^{\varphi}}\right]^{-\lambda}-1\right)^{-\gamma}\right\}^{-\alpha}\right)^{\beta}\right)\right. \\
& \left.\left.+\left(\delta_{2}+\delta_{3}\right)\left(1-2\left(1-\left\{1+\left(\left[1-e^{-\delta v^{\varphi}}\right]^{-\lambda}-1\right)^{-\gamma}\right\}^{-\alpha}\right)^{\beta}\right)\right\}\right\} \\
& \left\{1-\left(1-\left\{1+\left(\left[1-e^{-\delta u^{\varphi}}\right]^{-\lambda}-1\right)^{-\gamma}\right\}^{-\alpha}\right)^{\beta}\right. \\
& \times\left(1+\left(\delta_{1}+\delta_{3}\right)\left(1-\left\{1+\left(\left[1-e^{-\delta u^{\varphi}}\right]^{-\lambda}-1\right)^{-\gamma}\right\}^{-\alpha}\right)^{\beta}\right) \\
& -\left(1-\left\{1+\left(\left[1-e^{-\delta v^{\varphi}}\right]^{-\lambda}-1\right)^{-\gamma}\right\}^{-\alpha}\right)^{\beta} \\
& \times\left(1+\left(\delta_{2}+\delta_{3}\right)\left(1-\left\{1+\left(\left[1-e^{-\delta \nu^{\varphi}}\right]^{-\lambda}-1\right)^{-\gamma}\right\}^{-\alpha}\right)^{\beta}\right) \\
& +(\alpha \beta \gamma \lambda \delta \varphi)^{2}(u v)^{\varphi-1} e^{-\delta\left(u^{\varphi}+v^{\varphi}\right)}\left(\left[1-e^{-\delta u^{\varphi}}\right]\left[1-e^{-\delta v^{\varphi}}\right]\right)^{-(\lambda+1)} \\
& \times\left(\left(\left[1-e^{-\delta u^{\varphi}}\right]^{-\lambda}-1\right)\left(\left[1-e^{-\delta v^{\varphi}}\right]^{-\lambda}-1\right)\right)^{-(\gamma+1)} \\
& \times\left\{\left(1+\left(\left[1-e^{-\delta u^{\varphi}}\right]^{-\lambda}-1\right)^{-\gamma}\right)\left(1+\left(\left[1-e^{-\delta \nu^{\varphi}}\right]^{-\lambda}-1\right)^{-\gamma}\right)\right\}^{-(\alpha+1)} \\
& \times\left\{\left(1-\left\{1+\left(\left[1-e^{-\delta u^{\varphi}}\right]^{-\lambda}-1\right)^{-\gamma}\right\}^{-\alpha}\right)\right. \\
& \left.\times\left(1-\left\{1+\left(\left[1-e^{-\delta \varphi^{\varphi}}\right]^{-\lambda}-1\right)^{-\gamma}\right\}^{-\alpha}\right)\right\}^{\beta-1} \\
& \times\left\{1+\left(\delta_{1}+\delta_{3}\right)\left(1-2\left(1-\left\{1+\left(\left[1-e^{-\delta u^{\varphi}}\right]^{-\lambda}-1\right)^{-\gamma}\right\}^{-\alpha}\right)^{\beta}\right)\right. \\
& \left.\left.+\left(\delta_{2}+\delta_{3}\right)\left(1-2\left(1-\left\{1+\left(\left[1-e^{-\delta \varphi^{\varphi}}\right]^{-\lambda}-1\right)^{-\gamma}\right\}^{-\alpha}\right)^{\beta}\right)\right\}\right\}^{-1}
\end{aligned}
$$




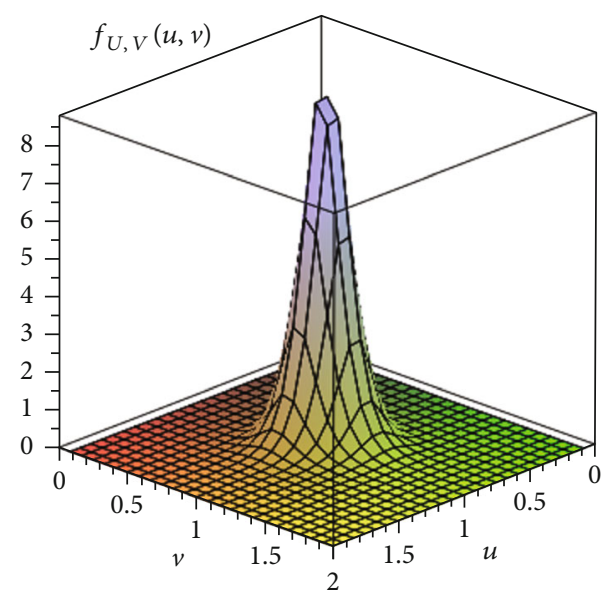

(a)

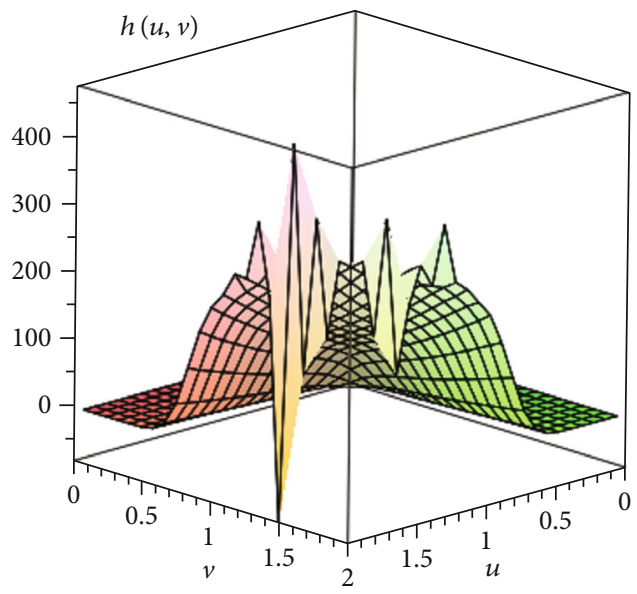

(c)

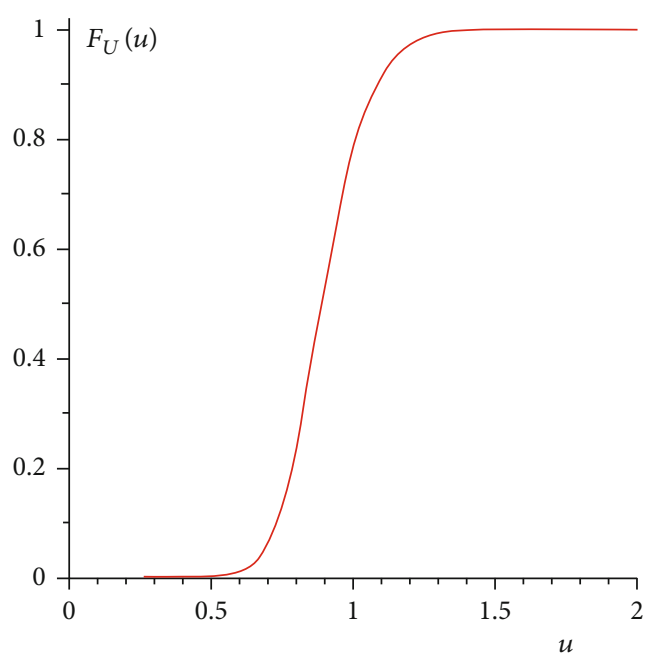

(e)

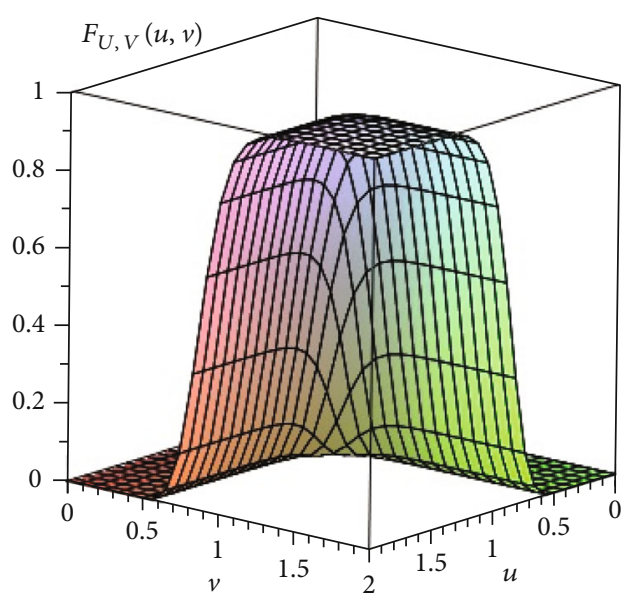

(b)

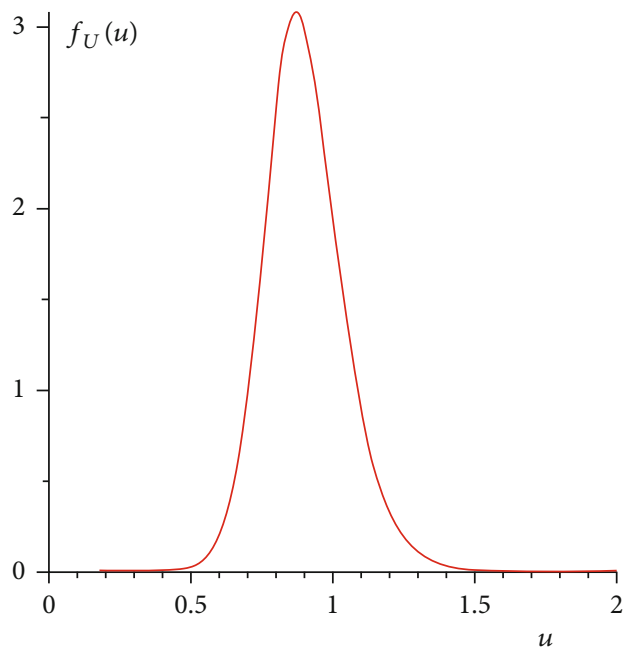

(d)

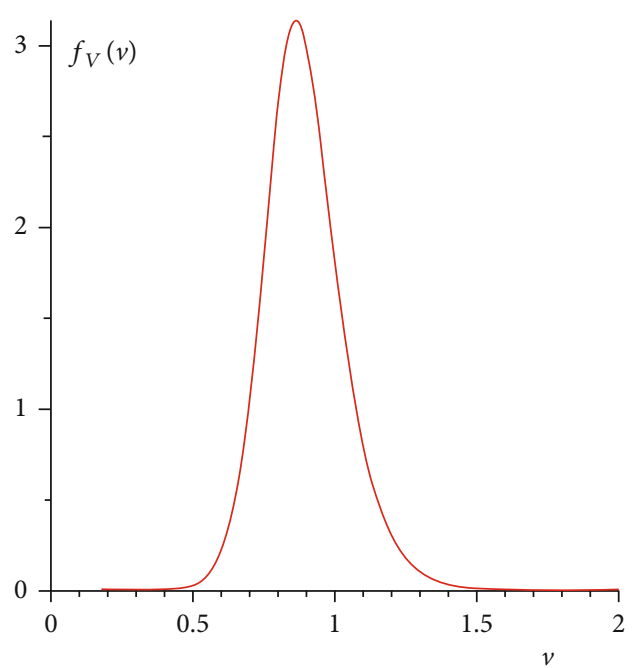

(f)

Figure 1: Continued. 


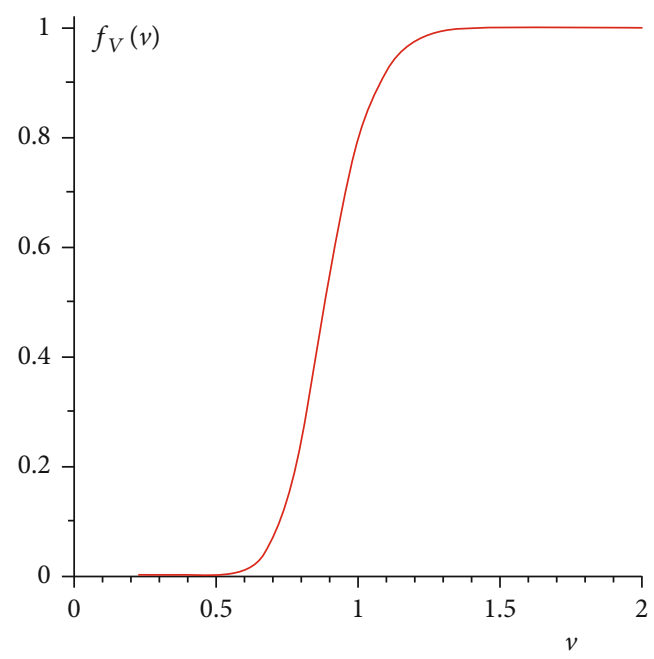

(g)

Figure 1: For the set of parameters $\alpha=1, \beta=1.8, \gamma=2.0, \lambda=1.5, \delta=1.6, \delta_{1}=-0.6, \delta_{2}=-0.5, \delta_{3}=0.7$, and $\phi=2.5$, the density function (4) is given in (a), the cumulative function (3) is given in (b), the hazard function (17) is given in (c), and the marginals (5), (7), (6), and (8) are given in $(\mathrm{d}-\mathrm{g})$, respectively.

\section{Estimation}

The maximum likelihood method is used to perform point estimators of the unknown parameters $\Theta=$ $\left(\alpha, \beta, \gamma, \lambda, \varphi, \delta, \delta_{1}, \delta_{2}, \delta_{3}\right)^{\prime}$. Let $\left(u_{1}, v_{1}\right),\left(u_{2}, v_{2}\right), \cdots,\left(u_{n}, v_{n}\right)$ be a random sample from the BIEGIKw-Weibull random variable. The maximum log-likelihood function is given by $L(\Theta)=L_{1}(\Theta)+L_{2}(\Theta)$, where

$$
\begin{aligned}
& L_{1}(\Theta)=2 n \ln (\alpha \beta \gamma \lambda \delta \varphi)+(\varphi-1) \sum_{i=1}^{n} \ln u_{i}-\delta \sum_{i=1}^{n} u_{i}^{\varphi}-(\lambda+1) \\
& \left.\times \sum_{i=1}^{n} \ln \left[1-e^{-\delta u_{i}^{\varphi}}\right]\right)-(\gamma+1) \sum_{i=1}^{n} \ln \left(\left[1-e^{-\delta u_{i}^{\varphi}}\right]^{-\lambda}-1\right) \\
& -(\alpha+1) \times \sum_{i=1}^{n} \ln \left\{1+\left(\left[1-e^{-\delta u_{i}^{\varphi}}\right]^{-\lambda}-1\right)^{-\gamma}\right\} \\
& +(\beta-1) \times \sum_{i=1}^{n} \ln \left(1-\left(\left\{1+\left(\left[\left(1-e^{-\delta u_{i}^{\varphi}}\right]^{-\lambda}-1\right)^{-\gamma}\right)\right\}^{-\alpha}\right)+,\right. \\
& L_{2}(\Theta)=(\varphi-1) \sum_{i=1}^{n} \ln v_{i}-\delta \sum_{i=1}^{n} v_{i}^{\varphi}-(\lambda+1) \sum_{i=1}^{n} \ln \left[1-e^{-\delta v_{i}^{\varphi}}\right] \\
& -(\gamma+1) \times \sum_{i=1}^{n} \ln \left(\left[1-e^{-\delta v_{i}^{\varphi}}\right]^{-\lambda}-1\right) \\
& -(\alpha+1) \sum_{i=1}^{n} \ln \left\{1+\left(\left[1-e^{-\delta v_{i}^{\varphi}}\right]^{-\lambda}-1\right)^{-\gamma}\right\} \\
& +(\beta-1) \sum_{i=1}^{n} \ln \left(1-\left\{1+\left(\left[1-e^{-\delta v_{i}^{\varphi}}\right]^{-\lambda}-1\right)^{-\gamma}\right\}^{-\alpha}\right) \\
& +\sum_{i=1}^{n} \ln \left\{1+\left(\delta_{1}+\delta_{3}\right)\left(1-2\left(1-\left\{1+\left(\left[1-e^{-\delta u_{i}^{\varphi}}\right]^{-\lambda}-1\right)^{-\gamma}\right\}^{-\alpha}\right)^{\beta}\right)\right. \\
& \left.+\left(\delta_{2}+\delta_{3}\right)\left(1-2\left(1-\left\{1+\left(\left[1-e^{-\delta v_{i}^{\varphi}}\right]^{-\lambda}-1\right)^{-\gamma}\right\}^{-\alpha}\right)^{\beta}\right)\right\} .
\end{aligned}
$$

Consider the score vector $\Psi=\left(\Psi_{\alpha}, \Psi_{\beta}, \Psi_{\gamma}, \Psi_{\lambda}, \Psi_{\varphi}, \Psi_{\delta}\right.$, $\left.\Psi_{\delta_{1}}, \Psi_{\delta_{2}}, \Psi_{\delta_{3}}\right)^{\prime}$, where $\Psi_{\alpha}=\Psi_{\alpha 1}+\Psi_{\alpha 2}, \Psi_{\beta}=\Psi_{\beta 1}+\Psi_{\beta 2}, \Psi_{\gamma}$ $=\Psi_{\gamma 1}+\Psi_{\gamma 2}, \Psi_{\lambda}=\Psi_{\lambda 1}+\Psi_{\lambda 2}, \Psi_{\varphi}=\Psi_{\varphi 1}+\Psi_{\varphi 2}+\Psi_{\varphi 3}, \Psi_{\delta}=$ $\Psi_{\delta 1}+\Psi_{\delta 2}$; then,

The system of equations can be solved via symbolic mathematical packages.

\section{Simulation}

In this section, simulation is performed for three different sets of parameters to verify our theoretical results and to discuss the new bivariate distribution properties. For the first set of parameters, $\alpha=1, \beta=1.8, \gamma=2.0, \lambda=1.5, \delta=1.6, \delta_{1}=-0.6, \delta_{2}=-$ $0.5, \delta_{3}=0.7$, and $\phi=2.5$. The symmetry, unimodality, and high beakness of the density function (4) can be observed in Figure $1(\mathrm{a})$. For $(u, v)$ approximately greater than $(2,2)$, the cumulative function (3) approaches 1 (Figure 1(b)). The bivariate hazard function increases with the increase of $(u$, $v$ ) as displayed in Figure 1(c). Symmetry of marginals and bell shape densities with beakness can be noted in Figures $1(\mathrm{~d})-1(\mathrm{~g})$. For the second set of parameters, $\alpha=1.5$ , $\beta=1.0, \gamma=0.7, \lambda=2.0, \delta=0.6, \delta_{1}=0.7, \delta_{2}=0.8, \quad \delta_{3}=-$ 0.5 , and $\phi=1.0$. The density function (4) is unimodal and has high beak (Figure 2(a)). For $(u, v)$ approximately greater than $(10,10)$, the cumulative function (3) approaches 1 (Figure 2(b)). The bivariate hazard function decreases with the increase of $(u, v)$ (Figure 2(c)). Unimodality and right tail property can be observed for marginals (Figures 2(d)$2(\mathrm{~g}))$. For the third set of parameters, $\alpha=0.5, \beta=0.7, \gamma=$ $0.9, \lambda=0.6, \delta=0.2, \delta_{1}=0.5, \delta_{2}=0.4, \delta_{3}=0.1$, and $\phi=0.3$. The density function (4) deformed and becomes an open surface (Figure 3(a)). The cumulative function (3) approaches 1 as $(u, v)$ approaches infinity (Figure 3(b)). The bivariate hazard function decreases with the increase of 


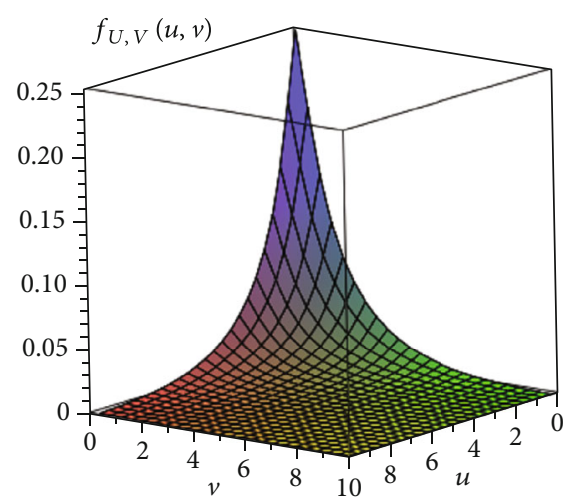

(a)

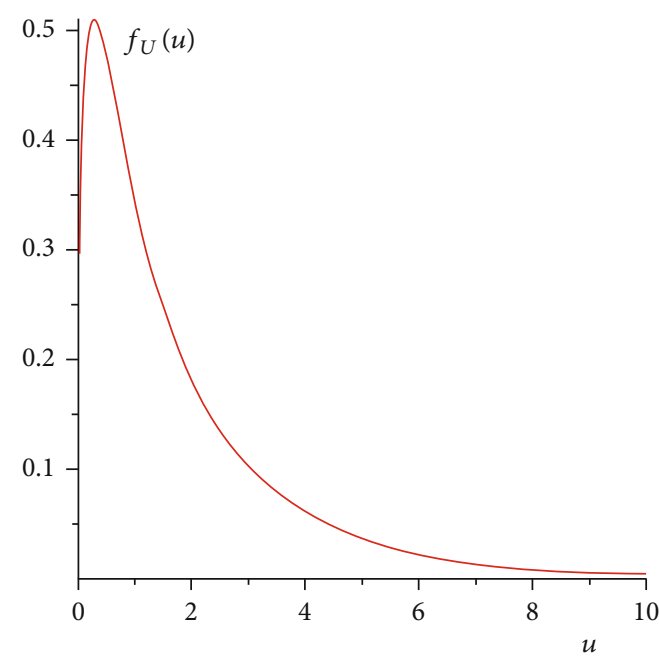

(d)

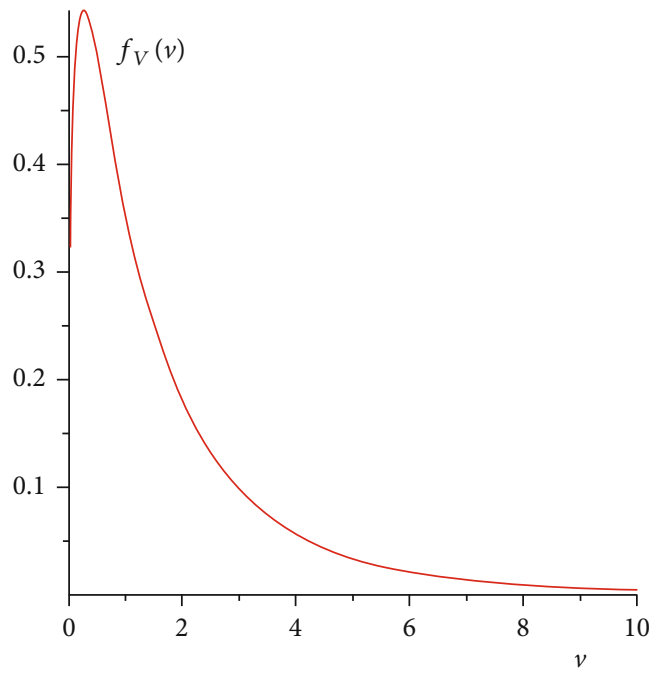

(f)

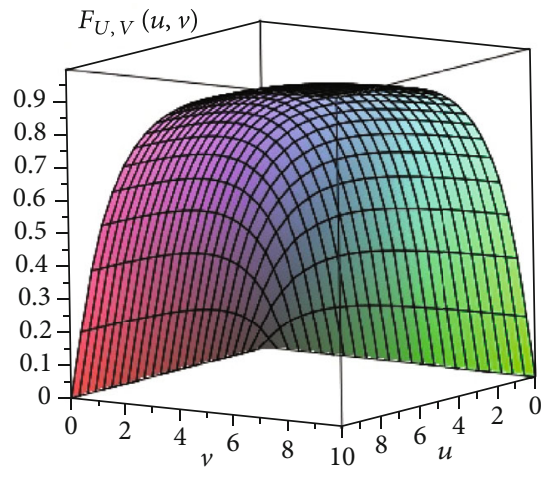

(b)

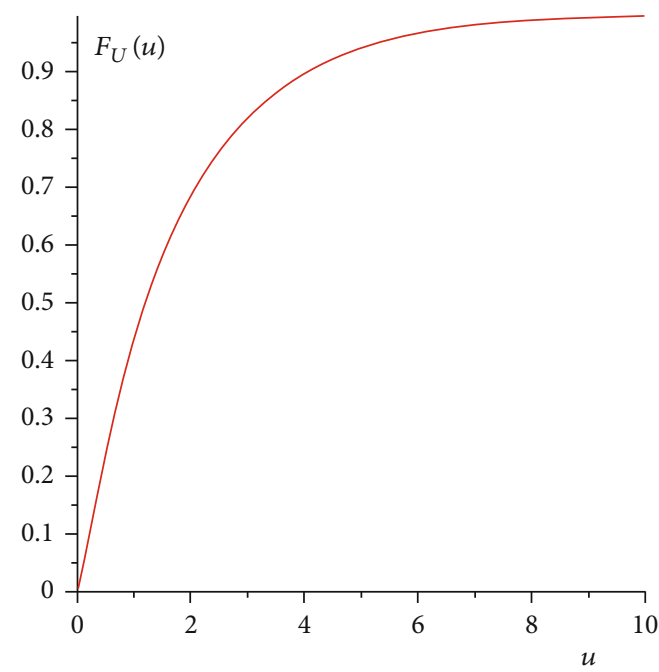

(e)

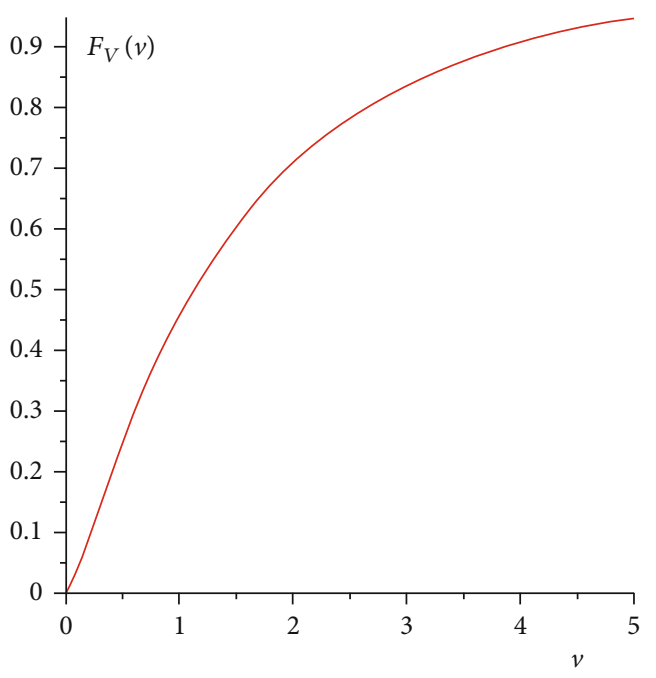

(g)

Figure 2: For the set of parameters $\alpha=1.5, \beta=1.0, \gamma=0.7, \lambda=2.0, \delta=0.6, \delta_{1}=0.7, \delta_{2}=0.8, \delta_{3}=-0.5$, and $\phi=1.0$, the density function (4) is given in (a), the cumulative function (3) is given in (b), the hazard function (17) is given in (c), and the marginals (5), (7), (6), and (8) are given in $(\mathrm{d}-\mathrm{g})$, respectively. 


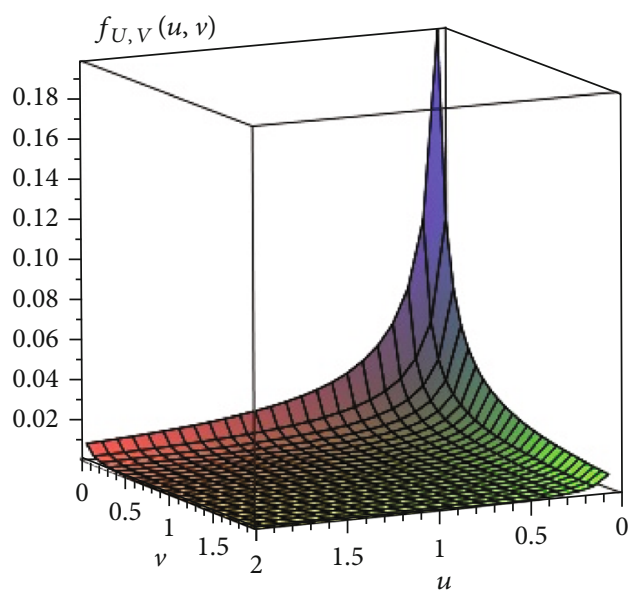

(a)

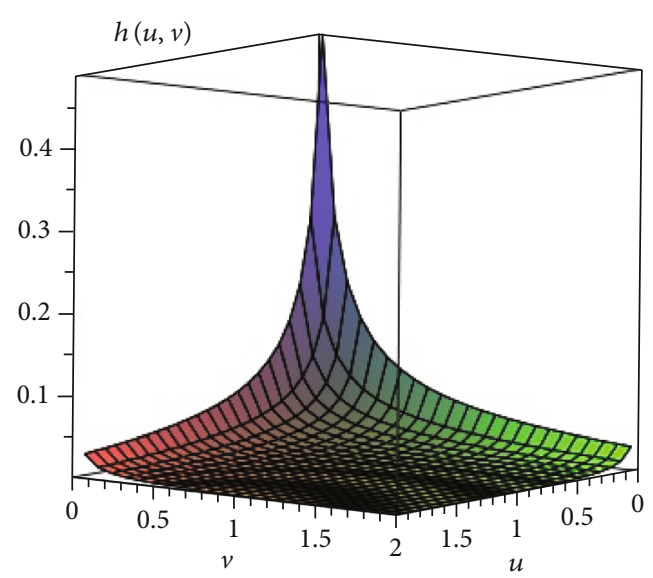

(c)

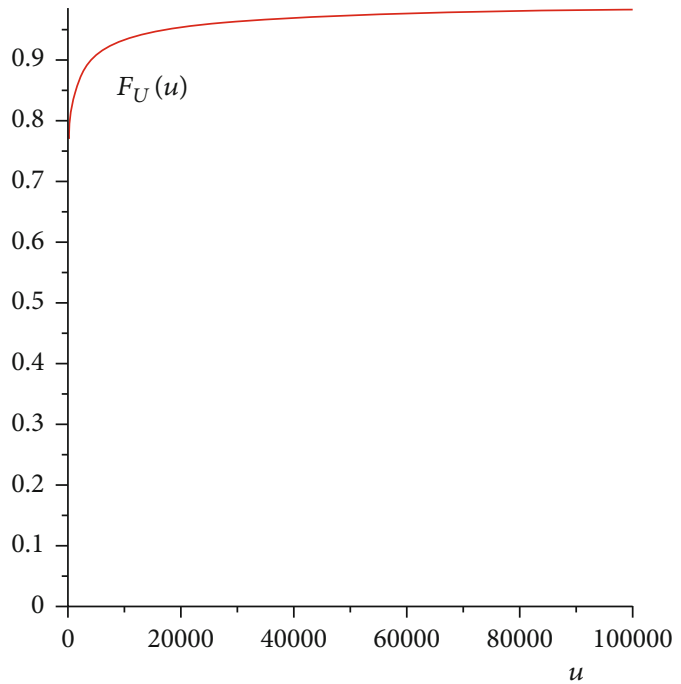

(e)

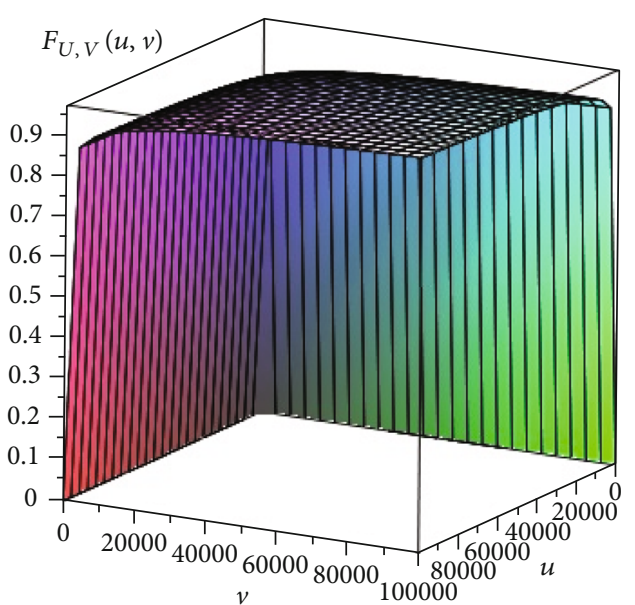

(b)

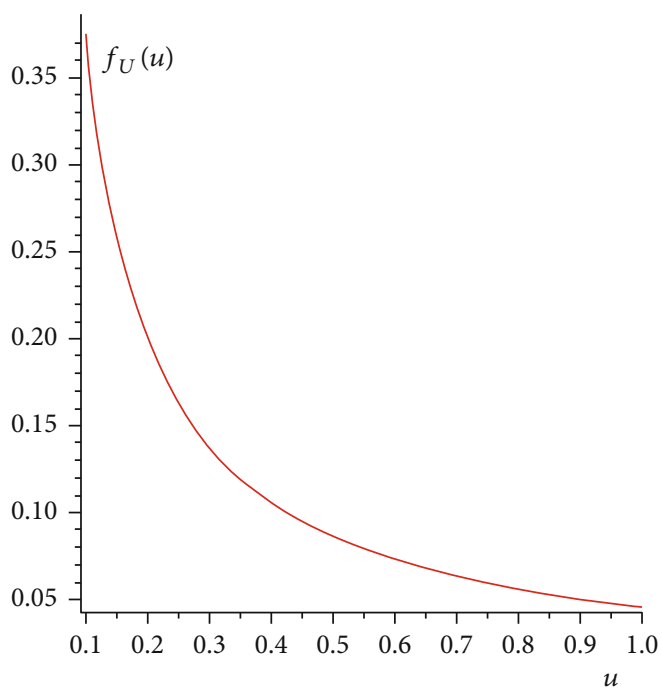

(d)

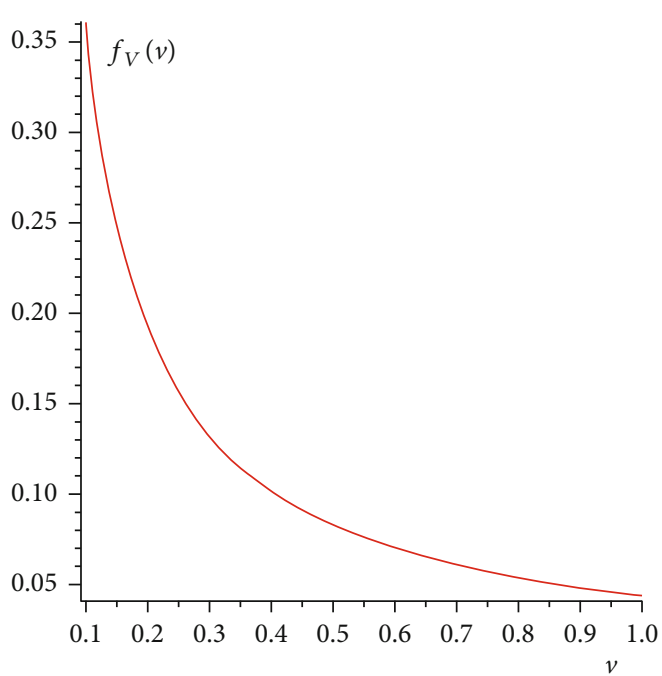

(f)

Figure 3: Continued. 


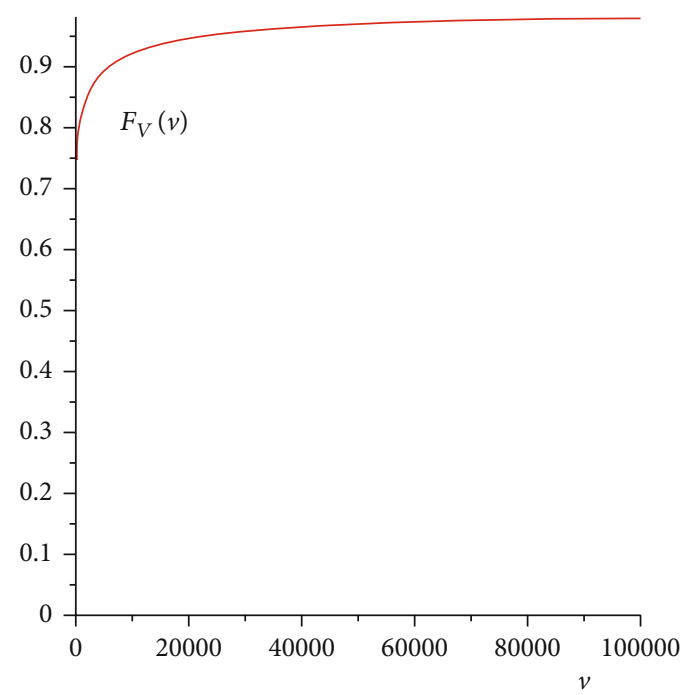

(g)

Figure 3: For the set of parameters $\alpha=0.5, \beta=0.7, \gamma=0.9, \lambda=0.6, \delta=0.2, \delta_{1}=0.5, \delta_{2}=0.4, \delta_{3}=0.1$, and $\phi=0.3$, the density function (4) is given in (a), the cumulative function (3) is given in (b), the hazard function (17) is given in (c), and the marginals (5), (7), (6), and (8) are given in $(\mathrm{d}-\mathrm{g})$, respectively.

TABle 1: Average maximum likelihood estimates for the first set of observations.

\begin{tabular}{|c|c|c|c|c|}
\hline Sample size & Parameter & Average estimate & Bias & SE \\
\hline \multirow{6}{*}{$n=20$} & $\alpha$ & 0.3420 & -0.158 & 0.0279 \\
\hline & $\beta$ & 2.510 & -0.49 & 0.134 \\
\hline & $\gamma$ & 0.535 & 0.035 & 0.102 \\
\hline & $\lambda$ & 0.573 & 0.073 & 0.118 \\
\hline & $\delta$ & 1.216 & -0.284 & 0.143 \\
\hline & $\varphi$ & 2.902 & -0.098 & 0.103 \\
\hline \multirow{6}{*}{$n=50$} & $\alpha$ & 0.4829 & -0.017 & 0.0914 \\
\hline & $\beta$ & 4.508 & 1.508 & 0.667 \\
\hline & $\gamma$ & 0.5422 & 0.042 & 0.0986 \\
\hline & $\lambda$ & 0.4006 & -0.099 & 0.0773 \\
\hline & $\delta$ & 0.858 & -0.642 & 0.153 \\
\hline & $\varphi$ & 2.801 & -0.199 & 0.177 \\
\hline \multirow{6}{*}{$n=100$} & $\alpha$ & 0.2661 & -0.244 & 0.0203 \\
\hline & $\beta$ & 3.206 & 0.206 & 0.243 \\
\hline & $\gamma$ & 0.9153 & 0.4153 & 0.0441 \\
\hline & $\lambda$ & 0.4187 & -0.0813 & 0.0748 \\
\hline & $\delta$ & 0.3047 & -0.1953 & 0.0325 \\
\hline & $\varphi$ & 3.1596 & 0.1596 & 0.0850 \\
\hline
\end{tabular}

$(u, v)$ (Figure 3(c)). Marginals are given in Figures 3(d)-3(g). Monte Carlo simulation is used to generate samples for different sizes to discuss the performance of the new distribution. The first set of observations is generated for the parameters
TABle 2: Average maximum likelihood estimates for the second set of observations.

\begin{tabular}{|c|c|c|c|c|}
\hline Sample size & Parameter & Average estimate & Bias & SE \\
\hline \multirow{6}{*}{$n=20$} & $\alpha$ & 1.463 & -0.237 & 0.185 \\
\hline & $\beta$ & 1.98 & 0.18 & 1.40 \\
\hline & $\gamma$ & 0.96 & -0.04 & 1.24 \\
\hline & $\lambda$ & 1.148 & 0.052 & 0.366 \\
\hline & $\delta$ & 1.991 & 0.391 & 0.561 \\
\hline & $\varphi$ & 0.883 & 0.183 & 0.240 \\
\hline \multirow{6}{*}{$n=50$} & $\alpha$ & 1.405 & -0.259 & 0.104 \\
\hline & $\beta$ & 2.424 & 0.624 & 0.956 \\
\hline & $\gamma$ & 1.39 & 0.39 & 1.21 \\
\hline & $\lambda$ & 1.497 & 0.297 & 0.579 \\
\hline & $\delta$ & 1.941 & 0.341 & 0.378 \\
\hline & $\varphi$ & 0.714 & -0.014 & 0.310 \\
\hline \multirow{6}{*}{$n=100$} & $\alpha$ & 1.448 & -0.251 & 0.0804 \\
\hline & $\beta$ & 1.363 & -0.437 & 0.332 \\
\hline & $\gamma$ & 1.08 & 0.08 & 1.76 \\
\hline & $\lambda$ & 1.403 & 0.203 & 0.309 \\
\hline & $\delta$ & 1.323 & -0.123 & 0.422 \\
\hline & $\varphi$ & 0.63 & -0.07 & 0.276 \\
\hline
\end{tabular}

$\alpha=0.5, \quad \beta=3, \gamma=0.5, \lambda=0.6, \delta=1.5, \delta_{1}=-0.7, \delta_{2}=-0.6$, $\delta_{3}=0.9$, and $\phi=3$; the second set is generated for the parameters $\alpha=1.7, \beta=1.8, \gamma=1.0, \lambda=1.2, \delta=1.6, \delta_{1}=-0.6, \delta_{2}=$ $-0.5, \delta_{3}=0.8$, and $\phi=0.7$. Considering $\delta_{1}, \delta_{2}$, and $\delta_{3}$ as 
TABLE 3: Simulated computer series system of two components.

\begin{tabular}{lccccccccccccccc}
\hline Sys & $\begin{array}{c}\text { Processor } \\
\text { lifetime }\end{array}$ & $\begin{array}{c}\text { Memory } \\
\text { lifetime }\end{array}$ & Sys & $\begin{array}{c}\text { Processor } \\
\text { lifetime }\end{array}$ & $\begin{array}{c}\text { Memory } \\
\text { lifetime }\end{array}$ & Sys & $\begin{array}{c}\text { Processor } \\
\text { lifetime }\end{array}$ & $\begin{array}{c}\text { Memory } \\
\text { lifetime }\end{array}$ & Sys & $\begin{array}{c}\text { Processor } \\
\text { lifetime }\end{array}$ & $\begin{array}{c}\text { Memory } \\
\text { lifetime }\end{array}$ & $\begin{array}{c}\text { Sys } \\
\text { Processor } \\
\text { lifetime }\end{array}$ & $\begin{array}{c}\text { Memory } \\
\text { lifetime }\end{array}$ \\
\hline 1 & 1.9292 & 3.9291 & 11 & 1.9386 & 4.0043 & 21 & 1.1739 & 3.3857 & 31 & 0.1181 & 0.0884 & 41 & 0.6270 & 1.7289 \\
2 & 3.6621 & 0.0026 & 12 & 2.1000 & 2.0513 & 22 & 1.3482 & 1.9705 & 32 & 5.0533 & 2.3238 & 42 & 0.7947 & 0.7947 \\
3 & 3.9608 & 0.8323 & 13 & 0.9867 & 0.9867 & 23 & 3.0935 & 3.0935 & 33 & 1.6465 & 2.0197 & 43 & 0.5079 & 5.3535 \\
4 & 2.3504 & 3.3364 & 14 & 0.1837 & 0.1837 & 24 & 2.1396 & 2.1548 & 34 & 0.9096 & 0.6214 & 44 & 2.5913 & 2.5913 \\
5 & 1.0833 & 3.3059 & 15 & 1.3989 & 4.1268 & 25 & 1.3288 & 0.9689 & 35 & 1.7494 & 2.3643 & 45 & 2.5372 & 2.4923 \\
6 & 2.8414 & 1.8438 & 16 & 2.3757 & 2.7953 & 26 & 0.1115 & 0.1115 & 36 & 0.1058 & 0.1058 & 46 & 1.1917 & 0.0801 \\
7 & 0.3309 & 0.3309 & 17 & 3.5202 & 1.4095 & 27 & 0.8503 & 2.8578 & 37 & 0.4593 & 0.4593 & 47 & 1.5254 & 4.4088 \\
8 & 2.9884 & 1.5961 & 18 & 2.3364 & 0.1624 & 28 & 0.1955 & 0.1955 & 38 & 0.9938 & 1.7689 & 48 & 1.0986 & 1.0986 \\
9 & 0.5784 & 1.8795 & 19 & 0.8584 & 1.9556 & 29 & 0.4614 & 0.8584 & 39 & 5.7561 & 0.3212 & 49 & 1.0051 & 1.0051 \\
10 & 0.5520 & 0.5520 & 20 & 4.3435 & 1.0001 & 30 & 3.3887 & 1.9796 & 40 & 6.2950 & 1.0495 & 50 & 1.3640 & 1.3640 \\
\hline
\end{tabular}

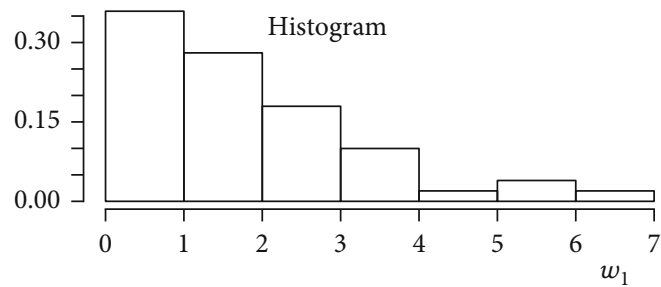

(a)

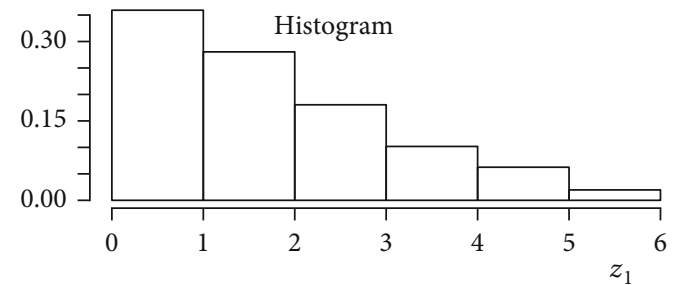

(b)

Figure 4: Histogram of the data in Table 3.

TABLE 4: The average maximum likelihood estimates of the BIEGIKw-Weibull parameters for the data in Table 3.

\begin{tabular}{lcc}
\hline Parameter & Estimate & AIC \\
\hline$\alpha$ & 0.82096924 & \\
$\beta$ & 0.38096869 & \\
$\lambda$ & 1.03547483 & \\
$\delta$ & 1.25168661 & 325.4836 \\
$\phi$ & 0.23330064 & \\
$\delta_{1}$ & 1.65658910 & \\
$\delta_{2}$ & -0.36575551 & \\
$\delta_{3}$ & 0.86741494 & \\
\hline
\end{tabular}




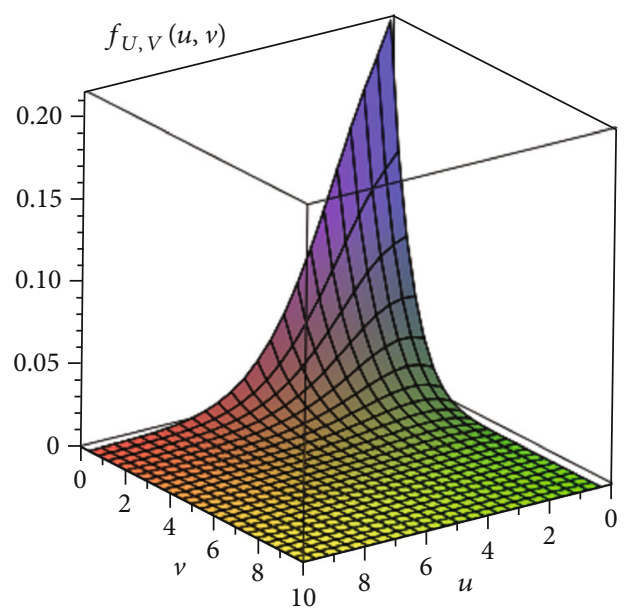

(a)

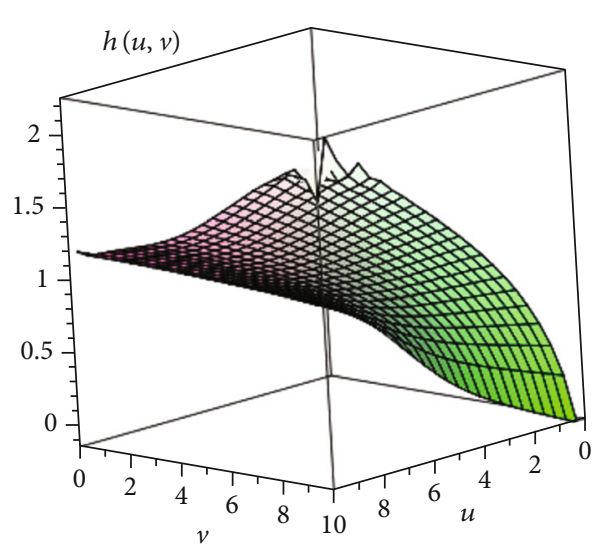

(c)

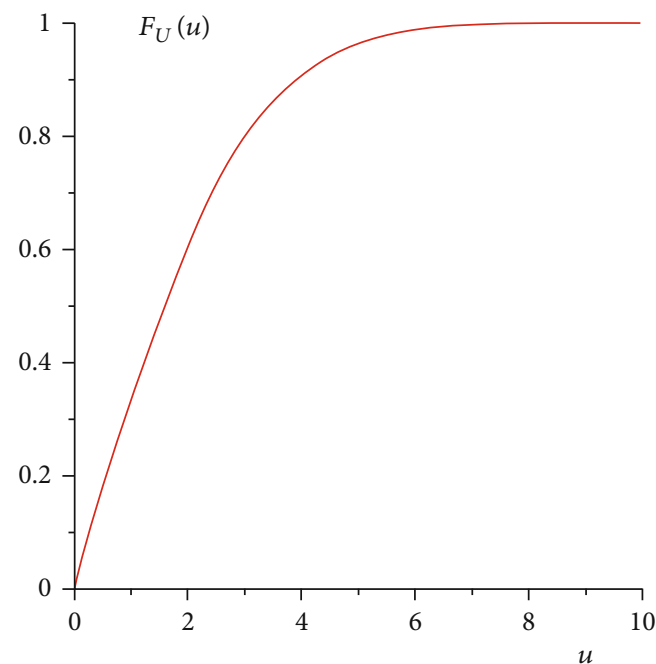

(e)

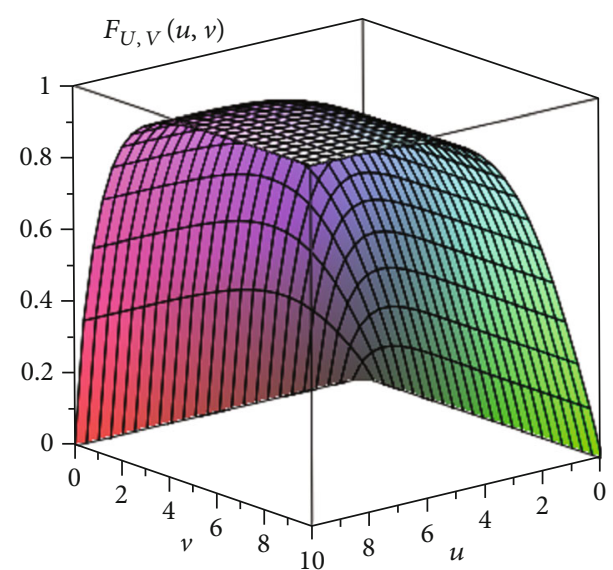

(b)

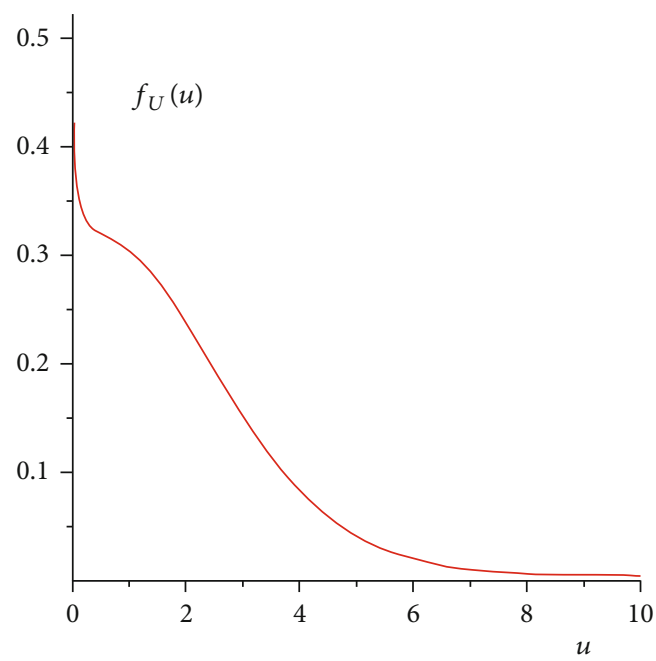

(d)

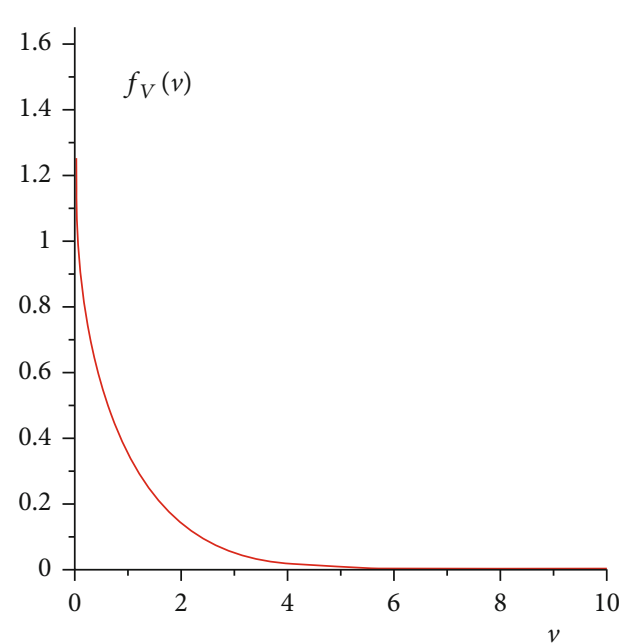

(f)

Figure 5: Continued. 


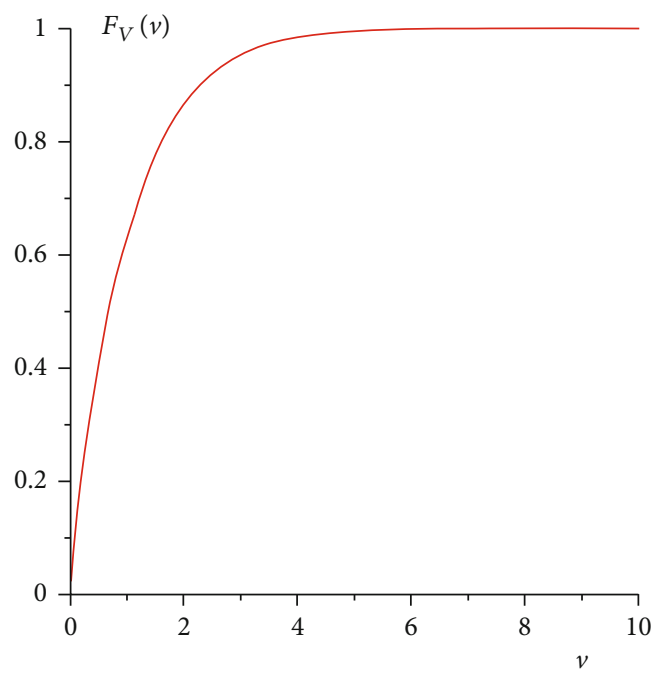

(g)

FIgure 5: (a) The joint density function, (b) the cumulative function, (c) the hazard function, and (d-g) the marginals for the estimated parameters in Table 4.

chosen parameters, the maximum likelihood method is used to estimate the rest parameters; results are given in Tables 1 and 2. Depending on the bias and standard error, the maximum likelihood method shows well performance.

\section{Real Data Application}

In this section, the data discussed by [31] is used to investigate the applicability of the new distribution. Let $\left(u_{1}, v_{1}\right),\left(u_{2}, v_{2}\right), \cdots,\left(u_{50}, v_{50}\right)$ be observed values of a BIEGIKw-Weibull random variable $(U, V)$ with parameters $\left(\alpha, \beta, \gamma, \lambda, \delta, \phi, \delta_{1}, \delta_{2}, \delta_{3}\right)$, where $U$ is the processor lifetime and $V$ is the memory lifetime; see Table 3 and Figure 4. The maximum likelihood method is conducted to estimate the parameters with AIC and BIC; see Table 4. The joint density function, hazard function, and marginals for the estimated parameters are given in Figure 5.

\section{Conclusions}

Analysis of correlated data is one of the most important problems in statistics and data science. Here, we introduced a new bivariate distribution named BIEGIKw-Weibull. The proposed model is with nine parameters. It is a flexible one. Theoretical properties including density function, cumulative function, marginals, copula function, conditional distributions, and conditional moments have been derived explicitly. The new model exhibits very rich characteristics that differ according to the parameters. That supports the applicability of the model for a large set of correlated data with various properties. Simulation clearly verified the theoretical properties and the richness of its preferable characteristics. For different values of the parameters, the distribution has extremely different properties that are clear from Figures 1-3. For a set of values, we can observe symmetry and close surface for density function side by side with unimodality (Figure 1). All these properties deformed for another set of parameters (Figure 3). The bivariate hazard function exhibited different shapes. Monte Carlo simulation and real data application prove the applicability of the new distribution and the availability of the maximum likelihood method.

\section{Data Availability}

The data used are included within the article.

\section{Conflicts of Interest}

The authors declare that there are no conflicts of interest regarding the publication of this article.

\section{Acknowledgments}

This project was funded by the Deanship of Scientific Research (DSR) at King Abdulaziz University (KAU), Jeddah, Saudi Arabia, under grant no. G: 80-351-1442. The authors therefore acknowledge with thanks DSR for the technical and financial support.

\section{References}

[1] A. Z. Keller and A. R. R. Kamath, "Alternative reliability models for mechanical systems," in Third International Conference in Reliability and Maintainability, Toulse, France, 1982.

[2] W. B. Nelson, Applied Life Data Analysis, Wiley, New Yourk, 1982.

[3] R. M. Pakungwati, Y. Widyaningsih, and D. Lestari, "Marshall-Olkin extended inverse Weibull distribution and its application," Journal of physics: conference series, vol. 1108, no. 1, article 012114, 2018.

[4] D. A. Ramadan and W. A. Magdy, "On the alpha-power inverse Weibull distribution," International Journal of Computer Applications, vol. 181, no. 11, pp. 6-12, 2018. 
[5] M. Q. Shahbaz, S. Shahbaz, and N. S. Butt, "The Kumaraswamy-inverse Weibull distribution," Pakistan Journal of Statistics and Operation Research, vol. 8, no. 3, pp. 479489, 2012.

[6] G. Aryal and I. Elbatal, "Kumaraswamy modified inverse Weibull distribution: theory and application," Applied Mathematics \& Information Sciences, vol. 9, no. 2, p. 651, 2015.

[7] M. A. Aldahlan, "The inverse Weibull inverse exponential distribution with application," International Journal of Contemporary Mathematical Sciences, vol. 14, no. 1, pp. 17-30, 2019.

[8] A. Aljouharah, "Estimating the parameters of an exponentiated inverted Weibull distribution under type-II censoring," Applied Mathematical Sciences, vol. 7, no. 35, pp. 1721-1736, 2013.

[9] A. S. Hassan and S. G. Nassr, "The inverse Weibull generator of distributions, properties and applications," Journal of Data Science, vol. 16, no. 4, pp. 723-742, 2018.

[10] S. A. Hassan, M. E. Almetwally, and M. G. Ibrahim, "Kumaraswamy inverted Topp-Leone distribution with applications to COVID-19 data," Computers, Materials \& Continua, vol. 68, no. 1, pp. 337-358, 2021.

[11] F. R. S. de Gusmão, E. M. Ortega, and G. M. Cordeiro, "The generalized inverse Weibull distribution," Statistical Papers, vol. 52, no. 3, pp. 591-619, 2011.

[12] A. A. L.-F. AM, A. A. El-Helbawy, and G. R. Al-Dayian, "Inverted Kumaraswamy distribution: properties and estimation," Pakistan Journal of Statistics, vol. 33, no. 1, pp. 37-67, 2017.

[13] Z. Iqbal, M. M. Tahir, N. Riaz, S. A. Ali, and M. Ahmad, "Generalized inverted Kumaraswamy distribution, properties and application," Open Journal of Statistics, vol. 7, no. 4, pp. 645662, 2017.

[14] F. Jamal, M. Arslan Nasir, G. Ozel, M. Elgarhy, and N. Mamode Khan, "Generalized inverted Kumaraswamy generated family of distributions: theory and applications," Journal of Applied Statistics, vol. 46, no. 16, pp. 2927-2944, 2019.

[15] H. Z. Muhammed, "On a bivariate generalized inverted Kumaraswamy distribution," Physica A: Statistical Mechanics and its Applications, vol. 553, article 124281, 2020.

[16] S. Mondal and D. Kundu, "A bivariate inverse Weibull distribution and its applications in complementary risks models," Journal of Applied Statistics, vol. 47, no. 6, pp. 1084-1108, 2021.

[17] J. A. Darwish and M. Q. Shahbaz, "Bivariate transmuted Burr distribution: properties and applcations," Pakistan Journal of Statistics \& Operation Research, vol. 17, no. 1, pp. 15-24, 2021.

[18] A. Sarhan and N. Balakrishnan, "A new class of bivariate distributions and its mixture," Journal of Multivariate Analysis, vol. 98, no. 7, pp. 1508-1527, 2007.

[19] D. Kundu and R. D. Gupta, "Bivariate generalized exponential distribution," Journal of Multivariate Analysis, vol. 100, pp. 581-593, 2009.

[20] D. Kundu and R. D. Gupta, "A class of bivariate models with proportional reversed hazard marginals," Sankhya B, vol. 72, pp. 236-253, 2010.

[21] A. Sarhan, D. C. Hamilton, B. Smith, and D. Kundu, "The bivariate generalized linear failure rate distribution and its multivariate extension," Computational Statistics and Data Analysis, vol. 55, no. 1, pp. 644-654, 2011.
[22] H. Z. Muhammed, "Bivariate inverse Weibull distribution," Journal of Statistical Computation and Simulation, vol. 86, no. 12, pp. 2335-2345, 2016.

[23] H. Z. Muhammed, "Bivariate Dagum distribution," International Journal of Reliability and Applications, vol. 18, no. 2, pp. 65-82, 2017.

[24] M. S. Eliwa and M. El-Morshedy, "Bivariate discrete inverse Weibull distribution,” 2018, https://arxiv.org/abs/1808.07748.

[25] H. Z. Muhammed, "Bivariate generalized Burr and related distributions: properties and estimation," Journal of Data Science, vol. 17, no. 3, pp. 532-548, 2019.

[26] M. Ganji, H. Bevraninand, and H. Golzar, "A new method for generating a continuous bivariate distribution families," Journal of The Iranian Statistical Society, vol. 17, no. 1, pp. 109129,2018

[27] A. Alzaatreh, C. Lee, and F. Famoye, "A new method for generating families of continuous distributions," Metron, vol. 71, pp. 63-79, 2013.

[28] Q. Ramzan, M. Amin, A. Elhassanein, and M. Ikram, "The extended generalized inverted Kumaraswamy Weibull distribution: properties and applications," AIMS Mathematics, vol. 6, no. 9, pp. 9955-9980, 2021.

[29] T. S. Durrani and X. Zeng, "Copulas for bivariate probability distributions," Electronics Letters, vol. 43, no. 4, 2007.

[30] J. Navarro, "Characterizations using the bivariate failure rate function," Statistics and Probability Letters, vol. 78, no. 12, p. 1349, 2010.

[31] R. P. Oliveira, J. A. Achcar, J. Mazucheli, and W. Bertoli, “A new class of bivariate Lindley distributions based on stress and shock models and some of their reliability properties," Reliability Engineering and System Safety, vol. 211, article 107528, 2021. 